\title{
Spin-polarized transport through a quantum dot: Anderson model with on-site Coulomb repulsion
}

\author{
N. Sergueev, ${ }^{1}$ Qing-feng Sun, ${ }^{1}$ Hong Guo, ${ }^{1,3}$ B. G. Wang, ${ }^{2}$ and Jian Wang ${ }^{2}$ \\ ${ }^{1}$ Center for the Physics of Materials and Department of Physics, McGill University, Montreal, PQ, Canada H3A 2T8 \\ ${ }^{2}$ Department of Physics, The University of Hong Kong, Pokfulam Road, Hong Kong, China \\ ${ }^{3}$ International Center of Quantum Structures, Institute of Physics, Chinese Academy of Sciences, Beijing, China
}

(Received 19 November 2001; published 4 April 2002)

\begin{abstract}
We report on a theoretical analysis of transport characteristics of a spin-valve system formed by a quantum dot connecting to two ferromagnetic electrodes whose magnetic moments are oriented at an angle $\theta$ with respect to each other. We pay special attention to the effects of a finite on-site Coulomb repulsion $U$. Using the Keldysh nonequilibrium Green's functions we derived a formula for the current in general terms of bias, temperature, and the parameters $\theta, U$. We have studied the local density of states and nonlinear conductance of this device in the Kondo regime at different polarization angle $\theta$. Our results suggest that the Kondo peaks in the local density of states and in the conductance can be modulated by $\theta$.
\end{abstract}

DOI: 10.1103/PhysRevB.65.165303

PACS number(s): 72.15.Qm, 85.75.Mm, 73.23.Hk, 73.63.-b

\section{INTRODUCTION}

Due to advances in materials science and nanofabrication techniques, magnetoelectronics and spin-electronics have become a realistic possibility and generated considerable recent excitement. ${ }^{1}$ In these systems, coherent charge as well as polarized spin are both utilized for electronic device function. ${ }^{1}$ The well-known giant magnetoresistance effect (GMR) is such a spin-polarized electronic transport effect. ${ }^{2,3}$ GMR system can be fabricated by sandwiching a nonmagnetic metal layer between two magnetic layers, ${ }^{2}$ and the electric current flowing through is varied by the relative orientation of the magnetic moments of the magnetic layers. Other practical variations of this structure can produce different device functions including the spin-valve transistor, ${ }^{4}$ the spin selective electron interferometer, ${ }^{5}$ and nonvolatile random access memory (RAM). ${ }^{6}$ Another interesting and important effect for spin-polarized transport is the tunneling magnetoresistance (TMR). A TMR device is usually presented by combinations of an insulating (I) material layer sandwiched in between two ferromagnetic (FM) layers, ${ }^{7}$ forming a FM/ I/FM tunneling structure. TMR devices have also shown sensitive magnetoresistance behavior at room temperature, ${ }^{8}$ and one of the particular attractions of a TMR device is that it carries lower current than the metallic GMR system which is a helpful device characteristic.

The high magnetoresistance in a TMR device is due to the spin-valve effect by which the resistance is different depending on whether the magnetization of the two FM metals are in parallel or antiparallel. Therefore, by rotating the magnetic moment of one FM metal relative to the other, the current is modulated by the relative angle $\theta$ of the two magnetic moments. Due to its importance, there have been many theoretical investigations on TMR structures ${ }^{9-16}$ where various transport, structural, and device properties were examined. Extensions of the conventional FM/I/FM TMR systems to FM/NM/FM structures have also received attention, where $\mathrm{NM}$ is a nonmagnetic region such as a quantum well, ${ }^{17}$ a carbon nanotube, ${ }^{18,19}$ and even a composite structure ${ }^{20}$ of I/FM/I. Because the NM region has its own electronic structure which can be quite complicated, ${ }^{19,20}$ the FM/NM/FM devices are expected to have more interesting features in their transport properties. The purpose of this work is to further investigate the magnetotransport behavior of FM/ $\mathrm{NM} / \mathrm{FM}$ structures.

In particular, we investigate the quantum transport characteristics of a FM/quantum dot/FM device. This system is more complicated than those studied before because transport through quantum dots (QD's) can be dominated by the Coulomb blockade effect, and important electron correlation effects such as the Kondo effect may arise. The Kondo effect is a prototypical many-body correlation effect involving interactions between a localized spin and free electrons. ${ }^{21}$ It has also been observed in semiconductor quantum dots. ${ }^{22-24}$ For a QD coupled to two normal leads, the physical origin of the Kondo effect is now well understood. ${ }^{21,22,24,25}$ Consider a single spin degenerate level $\epsilon_{d}$ of the QD such that $\epsilon_{d}<\mu_{N}$ $<\epsilon_{d}+U$, where $\mu_{N}$ is the chemical potential of the leads and $U$ the on-site $e-e$ interaction energy. An electron occupying $\epsilon_{d}$ cannot tunnel out of the QD because $\epsilon_{d}<\mu_{N}$; at the same time an electron outside the QD cannot tunnel into it unless the on-site Coulomb energy $U$ is overcome. This is the Coulomb blockade effect by which the first-order tunneling process is blockaded and no current flows through the QD. However, due to the Heisenberg uncertainty principle, there are virtual higher-order co-tunneling events which can still take place $e^{21,22,24}$ by which the electron inside a QD tunnels out followed by an electron with opposite spin tunneling into the $\mathrm{QD}$, on a time scale $\sim \hbar /\left|\mu_{N}-\epsilon_{d}\right|$. As a consequence, the local spin is flipped. At low temperatures, the coherent superposition of all possible co-tunneling events gives rise to the Kondo effect in which the time-averaged spin in the QD is zero due to frequent spin flips: the whole system, QD plus leads, forms a spin singlet, and a very narrow Kondo peak located at $\mu_{N}$ arises in the local density of states (LDOS). ${ }^{26}$ For a QD connected to FM leads, spin-polarized electrons are injected and it is therefore interesting to investigate the Kondo effect in such a FM/QD/FM device.

In this work, we will focus on two questions: (i) how is the QD Kondo effect influenced by the spin-valve behavior and vice versa? (ii) what is the nonlinear conductance characteristics of such an interacting TMR device? In our theory, 


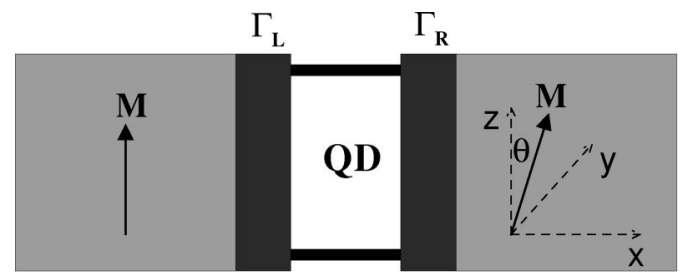

FIG. 1. Schematic plot showing the TMR device considered in this work. The quantum dot (QD) is contacted by two magnetic leads through the tunneling barriers. The magnetic moments of the leads are oriented an angle $\theta$ from each other, and the QD-leads couplings are $\Gamma_{L}$ and $\Gamma_{R}$.

the Coulomb repulsion is described by the Anderson model. ${ }^{27}$ It is well known that the Anderson model for a Kondo impurity also describes the physics of a quantum dot. There have been several experimental measurements on the discrete spectrum of a single QD, probed by transport ${ }^{28,29}$ and by capacitance spectroscopy, ${ }^{30}$ and theoretically Anderson's model is found to give results in good consistency with these experiments in and out of equilibrium. So far, this model has been applied to normal systems-QD's connected to nonmagnetic metallic leads which are easily biased to nonequilibrium and the QD potential is controlled by a gate voltage. ${ }^{31}$ For a QD device subjected to an external bias voltage, the interaction potential can be an important factor determining transport characteristics in the nonlinear regime. In fact, it may even be more important for devices with FM leads. Previous analysis of the bias dependence of TMR has neglected these interactions. ${ }^{9,17,20}$ Our investigations found that Kondo peaks in the FM/QD/FM device can be modulated by the magnetization angle $\theta$, while the current and nonlinear conductance also depend on the interaction parameter $U$.

The rest of the paper is organized as follows. In the next section we derive the formula for the current through the interacting TMR system. Section III presents numerical calculations of the local density of states and the nonlinear conductance. Section IV is a short summary. Some tedious algebra is included in the Appendix.

\section{THEORETICAL FORMULATION}

The TMR device we examine is schematically shown in Fig. 1. It consists of a quantum dot on which there is a Coulomb interaction of energy scale $U$, and the QD is connected to two ferromagnetic electrodes to the outside world. The magnetic moment $\mathbf{M}$ of the left electrode is pointing to the $z$ direction, the electric current is flowing in the $x$ direction, while the moment of the right electrode is at an angle $\theta$ to the $z$ axis in the $y-z$ plane. In second quantized form this device is described by the following Hamiltonian:

$$
H=H_{L}+H_{R}+H_{d o t}+H_{T} .
$$

$H_{d o t}$ describes the quantum dot including the Coulomb interaction represented by a finite $U$ Anderson term,

$$
\begin{aligned}
H_{d o t}= & \sum_{n \sigma} \epsilon_{n} d_{n \sigma}^{+} d_{n \sigma} \\
& +U \sum_{n, n^{\prime}, \sigma, \sigma^{\prime}\left(n \sigma \neq n^{\prime} \sigma^{\prime}\right)} d_{n \sigma}^{+} d_{n \sigma} d_{n^{\prime} \sigma^{\prime}}^{+} d_{n^{\prime} \sigma^{\prime}} .
\end{aligned}
$$

$H_{L}$ and $H_{R}$ describe the left and right electrodes where a dc bias potential is applied, and $H_{T}$ models the coupling between electrodes and the quantum dot region (the scattering region):

$$
H_{\alpha}=\sum_{k \sigma}\left[\left(\epsilon_{k \alpha}+\sigma M\right) c_{k \alpha \sigma}^{+} c_{k \alpha \sigma}\right]=\sum_{k \sigma} \epsilon_{k \alpha \sigma} c_{k \alpha \sigma}^{+} c_{k \alpha \sigma},
$$

and

$$
\alpha=L, R,
$$

$$
\begin{aligned}
H_{T}= & \sum_{n, k \sigma}\left[T_{k L \sigma} c_{k L \sigma}^{+} d_{n \sigma}+T_{k R \sigma}\left(\cos \frac{\theta}{2} c_{k R \sigma}^{+}\right.\right. \\
& \left.\left.-\sigma \cdot \sin \frac{\theta}{2} c_{k R \sigma}^{+}\right) d_{n \sigma}+\text { c.c. }\right] .
\end{aligned}
$$

In the model above, ${ }^{32} \epsilon_{k \alpha}$ is the energy of conduction electrons in the $\alpha$ electrode and is characterized by index $k$ $=|\mathbf{k}|$, where $\mathbf{k}$ is the wave vector. The operator $c_{k \alpha \sigma}^{+}\left(c_{k \alpha \sigma}\right)$ creates (annihilates) a conduction electron with spin index $\sigma$ inside the $\alpha$ electrode. Similarly, $d_{n \sigma}^{+}\left(d_{n \sigma}\right)$ is the creation (annihilation) operator of electrons with spin $\sigma$ at energy level $n$ for the quantum dot region. Although we can assume a multilevel QD with levels at $\epsilon_{n}$, for simplicity in the following we will consider just one level $\epsilon_{0}$, and the spin-orbit and multiplet splittings are neglected. In our notations, we have made another simplification that the value of molecular field $M$ is the same for the two electrodes and the spin-valve effect can be obtained ${ }^{33}$ by varying the angle $\theta$. In reality, $M$ shows the difference of density of states (DOS) between spin-up and spin-down electrons in the electrodes ${ }^{33}$ and are therefore different for different FM materials. However, we neglect such a detail because it will not alter any qualitative results of this work. With the model Hamiltonian Eqs. (1)(4), we now proceed to derive expressions for the transport current and the associated Green's functions.

The electric current flowing through the TMR device can be calculated ${ }^{34}$ in terms of the Green's functions of the QD:

$I_{\alpha}=\frac{i e}{\hbar} \int \frac{d \omega}{2 \pi} \operatorname{Tr}\left(\boldsymbol{\Gamma}_{\alpha}(\omega)\left\{\mathbf{G}^{<}(\omega)+f_{\alpha}(\omega)\left[\mathbf{G}^{R}(\omega)-\mathbf{G}^{A}(\omega)\right]\right\}\right)$,

where $f_{\alpha} \equiv f\left(\omega-\mu_{\alpha}\right)$ is the Fermi function of the $\alpha$ lead, and the trace is over both the state index and spin index. Here

$$
\Gamma_{\alpha}(\omega)=\left(\begin{array}{ll}
\Gamma_{\alpha \uparrow}(\omega) & 0 \\
0 & \Gamma_{\alpha \downarrow}(\omega)
\end{array}\right),
$$

with $\Gamma_{\alpha \sigma}(\omega)=2 \pi \sum_{k \in \alpha}\left|T_{k \alpha \sigma}\right|^{2} \delta\left(w-\epsilon_{k \alpha \sigma}\right)$ the linewidth function. The matrix $T_{k \alpha \sigma}$ describes coupling to the leads; $\mathbf{G}^{R}(\omega, U)$ is a $2 \times 2$ matrix in spin space for the retarded Green's function with $\mathbf{U}$ the on-site Coulomb repulsion. If 
one neglects the strong $e-e$ interaction, i.e., in Hartree approximation, $\mathbf{G}^{R}(\omega, U)$ is typically written as

$$
\mathbf{G}^{R}(\omega, U)=\frac{1}{\omega-\mathbf{H}_{d o t}-\mathbf{\Sigma}^{R}},
$$

where the self-energy $\boldsymbol{\Sigma}^{R}$ is also a $2 \times 2$ matrix in spin space and has contributions from both leads, $\boldsymbol{\Sigma}^{R}=\boldsymbol{\Sigma}_{L}^{R}(\omega)$ $+\boldsymbol{\Sigma}_{R}^{R}(\omega)$. $\boldsymbol{\Sigma}^{R}$ describes coupling of the QD region to the two magnetic electrodes. For a strongly interacting QD, on the other hand, we will derive $\mathbf{G}^{R}(\omega)$ in the following with details summarized in the Appendix.

In Eq. (5) $\mathbf{G}^{<}(\omega)$ is the lesser Green's function which is calculated ${ }^{34}$ through the Keldysh equation $\mathbf{G}^{<}=\mathbf{G}^{R} \mathbf{\Sigma}^{<} \mathbf{G}^{A}$. When there is no interaction, $\boldsymbol{\Sigma}^{<}(\omega)=\mathbf{\Sigma}_{0}^{<}(\omega)$ can be computed exactly and expressed in terms of the retarded and advanced self-energies as $\boldsymbol{\Sigma}_{0}^{<}(\omega)=-\Sigma_{\alpha \in L, R}\left[\boldsymbol{\Sigma}_{0 \alpha}^{R}(\omega)\right.$ $\left.-\boldsymbol{\Sigma}_{0 \alpha}^{A}(\omega)\right] f_{\alpha}(\omega)$. Here $\boldsymbol{\Sigma}_{0 \alpha}^{R}(\omega)\left[\boldsymbol{\Sigma}_{0 \alpha}^{A}(\omega)\right]$ is the retarded (advanced) noninteracting self-energy. However, when there is interaction as in our case, it is not straightforward to calculate the lesser self-energy. We therefore apply the ansatz proposed by $\mathrm{Ng}$ (Ref. 35) by assuming $\boldsymbol{\Sigma}^{<}=\boldsymbol{\Sigma}_{0}^{<} \mathbf{A}$ and $\boldsymbol{\Sigma}^{>}$ $=\boldsymbol{\Sigma}_{0}^{>} \mathbf{A}$, where $\mathbf{A}$ is a matrix to be determined by the condition $\boldsymbol{\Sigma}^{<}-\boldsymbol{\Sigma}^{>}=\boldsymbol{\Sigma}^{R}-\boldsymbol{\Sigma}^{A}$. This choice of the lesser selfenergy becomes exact in the noninteracting limit $U=0$. It is worth mentioning that this ansatz guarantees automatically the conservation of current. ${ }^{35}$ We obtain

$$
\boldsymbol{\Sigma}^{<}=\boldsymbol{\Sigma}_{0}^{<}\left(\boldsymbol{\Sigma}_{0}^{R}-\boldsymbol{\Sigma}_{0}^{A}\right)^{-1}\left(\boldsymbol{\Sigma}^{R}-\boldsymbol{\Sigma}^{A}\right)
$$

which is calculable from the knowledge of Green's functions and $\boldsymbol{\Sigma}_{0}$.

From Eq. (5), the current from the left lead to the QD region can be rewritten as

$$
I=\frac{i e}{\hbar} \int \frac{d \omega}{2 \pi} \operatorname{Tr}\left[\boldsymbol{\Gamma}_{L}\left(\mathbf{G}^{R} \mathbf{\Sigma} \mathbf{G}^{A} f_{L}+\mathbf{G}^{R} \boldsymbol{\Sigma}^{<} \mathbf{G}^{A}\right)\right],
$$

where $\boldsymbol{\Sigma} \equiv \boldsymbol{\Sigma}^{R}-\boldsymbol{\Sigma}^{A}$. Substituting Eq. (6) into Eq. (7), defining $\overline{\boldsymbol{\Gamma}}_{R} \equiv\left[f_{L} \mathbf{1}+\boldsymbol{\Sigma}_{0}^{<}\left(\boldsymbol{\Sigma}_{0}^{R}-\boldsymbol{\Sigma}_{0}^{A}\right)\right]\left(\boldsymbol{\Sigma}^{R}-\boldsymbol{\Sigma}^{A}\right)$ where $\mathbf{1}$ is a unit matrix, Eq. (7) is reduced to a compact and final form,

$$
I=\frac{i e}{\hbar} \int \frac{d \omega}{2 \pi} \operatorname{Tr}\left[\boldsymbol{\Gamma}_{L} \mathbf{G}^{R} \overline{\boldsymbol{\Gamma}}_{R} \mathbf{G}^{A}\right] .
$$

Although this formula looks similar to that without the onsite Coulomb interaction, it is important to realize that quantities inside the integrand are now functions of $U$.
The remaining task is to derive the $2 \times 2$ Green's function $G^{R}$ which appears in Eq. (8),

$$
\mathbf{G}^{R}=\left(\begin{array}{cc}
G_{\uparrow \uparrow}^{R} & G_{\uparrow \downarrow}^{R} \\
G_{\downarrow \uparrow}^{R} & G_{\downarrow \downarrow}^{R}
\end{array}\right) \equiv\left(\begin{array}{cc}
G_{11}^{R} & G_{12}^{R} \\
G_{21}^{R} & G_{22}^{R}
\end{array}\right),
$$

where the components are, by definition,

$$
G_{\sigma \sigma^{\prime}}^{R} \equiv\left\langle\left\langle d_{\sigma}, d_{\sigma^{\prime}}^{+}\right\rangle\right\rangle \equiv-i \int_{0}^{\infty} e^{i \omega t}\left\langle\left\{d_{\sigma}(t), d_{\sigma^{\prime}}^{+}(0)\right\}\right\rangle d t .
$$

Here, $\{$,$\} and \langle\rangle$ denote anticommutator and statistical average of operators, respectively. In this work we evaluate $G^{R}$ using the standard equation of motion method for which we refer interested readers to Refs. 31, and 35-38. In the following we outline only the essentials specific to the present system and we present some tedious algebra in the Appendix.

Iterating the equation of motion, ${ }^{31,35-38}$ we obtain

$$
\begin{aligned}
\left(\omega-\epsilon_{0}\right)\left\langle\left\langle d_{\sigma}, d_{\sigma^{\prime}}^{+}\right\rangle\right\rangle= & \delta_{\sigma \sigma^{\prime}}+\sum_{k} T_{k L \sigma}^{\star}\left\langle\left\langle c_{k L \sigma}, d_{\sigma^{\prime}}^{+}\right\rangle\right\rangle \\
& +\sum_{k} T_{k R \sigma}^{\star}\left\langle\left\langle c_{k R \sigma}, d_{\sigma^{\prime}}^{+}\right\rangle\right\rangle \cos \left(\frac{\theta}{2}\right) \\
& -\sigma \sum_{k} T_{k R \sigma}^{\star}\left\langle\left\langle c_{k R \bar{\sigma}}, d_{\sigma^{\prime}}^{+}\right\rangle\right\rangle \sin \left(\frac{\theta}{2}\right) \\
& +U \sum_{\bar{\sigma} \neq \sigma}\left\langle\left\langle d_{\bar{\sigma}}^{+} d_{\bar{\sigma}} d_{\sigma}, d_{\sigma^{\prime}}^{+}\right\rangle\right\rangle ; \\
\left(\omega-\epsilon_{k L \sigma}\right)\left\langle\left\langle c_{k L \sigma}, d_{\sigma^{\prime}}^{+}\right\rangle\right\rangle=T_{k L \sigma}\left\langle\left\langle d_{\sigma}, d_{\sigma^{\prime}}^{+}\right\rangle\right\rangle ; & \\
\left(\omega-\epsilon_{k R \sigma}\right)\left\langle\left\langle c_{k R \sigma}, d_{\sigma^{\prime}}^{+}\right\rangle\right\rangle= & T_{k R \sigma} \cos \left(\frac{\theta}{2}\right)\left\langle\left\langle d_{\sigma}, d_{\sigma^{\prime}}^{+}\right\rangle\right\rangle \\
& -\bar{\sigma} T_{k R \bar{\sigma}} \sin \left(\frac{\theta}{2}\right)\left\langle\left\langle d_{\sigma^{-}}, d_{\sigma^{\prime}}^{+}\right\rangle\right\rangle ; \\
\left(\omega-\epsilon_{k R \bar{\sigma}}\right)\left\langle\left\langle c_{k R \bar{\sigma}}, d_{\sigma^{\prime}}^{+}\right\rangle\right\rangle= & T_{k R \bar{\sigma}} \cos \left(\frac{\theta}{2}\right)\left\langle\left\langle d_{\bar{\sigma}}^{-}, d_{\sigma^{\prime}}^{+}\right\rangle\right\rangle \\
& -\sigma T_{k R \sigma} \sin \left(\frac{\theta}{2}\right)\left\langle\left\langle d_{\sigma^{\prime}}, d_{\sigma^{\prime}}^{+}\right\rangle\right\rangle ;
\end{aligned}
$$

$$
\begin{aligned}
& \left(\omega-\epsilon_{0}-U\right)\left\langle\left\langle d_{\bar{\sigma}}^{+} d_{\bar{\sigma}} d_{\sigma}, d_{\sigma^{\prime}}^{+}\right\rangle\right\rangle=\left\langle\left\{d_{\bar{\sigma}}^{+} d_{\bar{\sigma}} d_{\sigma}, d_{\sigma^{\prime}}^{+}\right\}\right\rangle+\sum_{k} T_{k L \sigma}^{\star}\left\langle\left\langle d_{\bar{\sigma}}^{+} d_{\bar{\sigma}}^{-} c_{k L \sigma}, d_{\sigma^{\prime}}^{+}\right\rangle\right\rangle-\sum_{k} T_{k L \bar{\sigma}}^{\star}\left\langle\left\langle d_{\bar{\sigma}}^{+} d_{\sigma} c_{k L \bar{\sigma}}, d_{\sigma^{\prime}}^{+}\right\rangle\right\rangle \\
& -\sum_{k} T_{k L \bar{\sigma}}\left\langle\left\langle c_{k L \bar{\sigma}}^{+} d_{\bar{\sigma}}^{-} d_{\sigma}, d_{\sigma^{\prime}}^{+}\right\rangle\right\rangle+\sum_{k} T_{k R \sigma}^{\star}\left\langle\left\langle d_{\bar{\sigma}}^{+} d_{\bar{\sigma}} c_{k R \sigma}, d_{\sigma^{\prime}}^{+}\right\rangle\right\rangle \cos \left(\frac{\theta}{2}\right)-\sigma \sum_{k} T_{k R \sigma}^{\star}\left\langle\left\langle d_{\bar{\sigma}}^{+} d_{\bar{\sigma}} c_{k R \bar{\sigma}}, d_{\sigma^{\prime}}^{+}\right\rangle\right\rangle \\
& \times \sin \left(\frac{\theta}{2}\right)-\sum_{k} T_{k R \bar{\sigma}}^{\star}\left\langle\left\langle d_{\bar{\sigma}}^{+} d_{\sigma} c_{k R \bar{\sigma}}, d_{\sigma^{\prime}}^{+}\right\rangle\right\rangle \cos \left(\frac{\theta}{2}\right)+\bar{\sigma} \sum_{k} T_{k R \bar{\sigma}}^{\star}\left\langle\left\langle d_{\bar{\sigma}}^{+} d_{\sigma} c_{k R \sigma}, d_{\sigma^{\prime}}^{+}\right\rangle\right\rangle \\
& \times \sin \left(\frac{\theta}{2}\right)-\sum_{k} T_{k R \bar{\sigma}}\left\langle\left\langle c_{k R \bar{\sigma}}^{+} d_{\bar{\sigma}}^{-} d_{\sigma}, d_{\sigma^{\prime}}^{+}\right\rangle\right\rangle \cos \left(\frac{\theta}{2}\right)+\bar{\sigma} \sum_{k} T_{k R \bar{\sigma}}\left\langle\left\langle c_{k R \sigma}^{+} d_{\bar{\sigma}} d_{\sigma}, d_{\sigma^{\prime}}^{+}\right\rangle\right\rangle \sin \left(\frac{\theta}{2}\right) .
\end{aligned}
$$


At this level, applying the Hartree-Fock decoupling approximation to Eq. (14) gives a solution ${ }^{38}$ for the Green's function. Such a solution predicts a local density of states (LDOS) which exhibits two peaks near $\epsilon_{0}$ and $\epsilon_{0}+U$, the former indicates a resonance transmission through the QD while the latter describes the Coulomb peak. In order to investigate how the Kondo resonance is affected by the spin valve, we consider the equation of motion for the Green's functions appearing on the right-hand side of Eq. (14), and we apply the following decoupling approximation:

$$
\begin{gathered}
\left\langle\left\langle c_{k \alpha \sigma}^{+} c_{k_{1} \alpha_{1} \sigma_{1}} d_{\sigma}, d_{\sigma^{\prime}}^{+}\right\rangle\right\rangle \approx \\
=\left\langle c_{k \alpha \sigma}^{+} c_{k_{1} \alpha_{1} \sigma_{1}}\right\rangle\left\langle\left\langle d_{\sigma}, d_{\sigma^{\prime}}^{+}\right\rangle\right\rangle \\
=\delta_{k k_{1}} \delta_{\alpha \alpha_{1}} \delta_{\sigma \sigma_{1}}\left\langle c_{k \alpha \sigma}^{+} c_{k \alpha \sigma}\right\rangle \\
\times\left\langle\left\langle d_{\sigma}, d_{\sigma^{\prime}}^{+}\right\rangle\right\rangle ; \\
\left\langle\left\langle d_{\sigma}^{+} c_{k \alpha \sigma} c_{k_{1} \alpha_{1} \sigma_{1}}, d_{\sigma^{\prime}}^{+}\right\rangle\right\rangle \approx 0, \\
\left\langle c_{k \alpha \sigma}^{+} d_{\sigma}\right\rangle=\left\langle d_{\sigma}^{+} c_{k \alpha \sigma}\right\rangle \approx 0 .
\end{gathered}
$$

This decoupling approximation has been known to give qualitatively correct Kondo physics at $T \leqslant T_{K} \cdot{ }^{39}$ For $T$ $>T_{K}$, it is also quantitative reasonable. ${ }^{38}$ With the decoupling, Eq. (14) is simplified and the Green's function in Eq. (10) can be derived explicitly as shown in the Appendix. The final result is

$$
\begin{gathered}
\left\langle\left\langle d_{\sigma}, d_{\sigma}^{+}\right\rangle\right\rangle=\frac{1+U Y_{\sigma} A C-U Y_{\sigma} A \frac{C^{\prime} D}{\bar{B}}}{B-\frac{D \bar{D}}{\bar{B}}}, \\
\left\langle\left\langle d_{\bar{\sigma}}, d_{\sigma}^{+}\right\rangle\right\rangle=-U Y_{\bar{\sigma}} A \frac{C^{\prime}}{B}+\frac{\bar{D}}{\bar{B}}\left\langle\left\langle d_{\sigma}, d_{\sigma}^{+}\right\rangle\right\rangle,
\end{gathered}
$$

where all the quantities on the right-hand side are known functions of energy and parameters $\theta, U$. They are given in Eqs. (A19), (A20), and (A25)-(A28) in the Appendix. Equations (18) and (19) form the basis for further numerical calculations for the FM/QD/FM system which we present in the next section.

\section{NUMERICAL ANALYSIS}

To numerically evaluate Eqs. (18) and (19), we first obtain solutions for the statistical averages $\left\langle n_{\sigma}\right\rangle$ and $\left\langle d_{\sigma}^{+} d_{\sigma}^{-}\right\rangle$. These statistical averages can be written in the following way:

$$
\begin{gathered}
\left\langle n_{\bar{\sigma}}\right\rangle=\left\langle d_{\bar{\sigma}}^{+} d_{\bar{\sigma}}^{-}\right\rangle=\operatorname{Im} \int \frac{d \omega}{2 \pi}\left\langle\left\langle d_{\bar{\sigma}}^{-}, d_{\bar{\sigma}}^{+}\right\rangle\right\rangle^{<}, \\
\left\langle d_{\sigma}^{+} d_{\bar{\sigma}}\right\rangle=-i \int \frac{d \omega}{2 \pi}\left\langle\left\langle d_{\bar{\sigma}}, d_{\sigma}^{+}\right\rangle\right\rangle^{<} .
\end{gathered}
$$

These equations are self-consistent because the matrix elements of the right-hand side $\left\langle\left\langle d_{\bar{\sigma}} d_{\bar{\sigma}}^{+}\right\rangle\right\rangle^{<}$and $\left\langle\left\langle d_{\bar{\sigma}} d_{\sigma}^{+}\right\rangle\right\rangle^{<}$are actually lesser Green's functions and they can be expressed in terms of retarded and advanced Green's functions as discussed in the previous section. These equations are therefore solved iteratively. ${ }^{31}$ After obtaining the statistical averages, we then proceed to calculate all other quantities from Eqs. (A3)-(A14) and (A25)-(A28) without further difficulty.

For our FM/QD/FM system, we calculated the local density of states $\operatorname{LDOS}=-(1 / \pi) \operatorname{Im} \Sigma_{\sigma}\left(G_{\sigma \sigma}^{R}\right)$ at different polarization angles $\theta$ (units set by $\hbar=e=2 m=1$ ). For QD's with normal leads in the Kondo regime, there are three peaks in the LDOS, ${ }^{39,40,31,35}$ as a function of energy at zero bias. One peak is due to the intradot renormalized level $\epsilon_{0}$, one corresponds to the Coulomb peak at $\epsilon_{0}+U$, and finally the Kondo peak at the chemical potential of the leads. In our FM/QD/FM system, these three peaks are also obtained as expected.

In the following we focus on a small energy range around the Kondo peak. For the QD having a spin-degenerate level and at equilibrium (zero bias), the Kondo peak is at the chemical potential of the leads giving rise to a resonant transmission through the QD. ${ }^{24}$ If a bias voltage is applied, the Kondo peak splits to two located at the chemical potential of each lead and the peak heights are suppressed since the electrons dissipatively jump from the lead with high chemical potential to that with low potential through the QD. Figure 2(a) shows $\operatorname{LDOS}(\epsilon)$ for different orientations $\theta$ versus energy $\epsilon$. The two Kondo peaks at each chemical potential are clearly observed. When $\theta=0$, i.e., when magnetic moments of the two FM leads are parallel, LDOS is largest at all temperatures [inset of Fig. 2(a)]. When $\theta=\pi$ for which the moments are antiparallel, the LDOS is the smallest. Therefore the Kondo peak height is modulated by the relative orientation of the magnetization of the leads in similar manner as the magnetoresistance. ${ }^{33}$ Figure 2(b) shows LDOS at temperature $K_{B} T=0.005$ and $\theta=0$ for different polarizations of the FM leads, here $K_{B}$ is the Boltzmann constant. As usual, the polarization is defined as $P \equiv\left(\Gamma_{\alpha \uparrow}-\Gamma_{\alpha \downarrow}\right) /\left(\Gamma_{\alpha \uparrow}\right.$ $\left.+\Gamma_{\alpha \downarrow}\right)$, here we have assumed that the left and right FM leads have the same $P$. From Fig. 2(b), when $P=0$ the Kondo peak LDOS is at a minimum, and it increases as $P$ increases. This result suggests that the Kondo resonance is enhanced when materials with larger polarization is used for the FM leads. The inset of Fig. 2(b) shows the change of shape of Kondo resonance on temperature. Since the Kondo peak is induced by single electron excitations from the many-body ground state, as temperature increases, the weight of ground state decreases and therefore Kondo peak disappears.

Next, we investigate the behavior of the magnetoconductance. The conductance of the FM/QD/FM system is obtained by calculating the current flowing through one of the contacts between the leads and the QD, as given in Eq. (8). Calculation of current requires the lesser Green's function which was discussed in Sec. II but it actually follows immediately from LDOS. The zero-temperature current is then the integrated density of states weighted by couplings to the leads. In the following our aim is to study conductance at different polarization and orientation of magnetic moments in the Kondo regime. 
Figures 3(a) and 3(b) show differential conductance $G$ with different polarizations at $\theta=0$ and $\pi$, as a function of bias voltage. The two broad peaks represent the main resonance peak due to the QD level $\epsilon_{0}$ and the main charging peak due to $\epsilon_{0}+U$. The sharp peak at zero bias between the main peaks is the Kondo resonance. The conductance value of the main peaks is approximately independent of $P$ for $\theta$ $=0$, while it reduces significantly with $P$ for $\theta=\pi$. This behavior of main peaks is similar to that of the conventional noninteracting ( $U=0)$ TMR system. From the point of view of resonance tunneling through two barriers, $\theta=0$ means the two barrier heights are the same, therefore the resonance tunneling probability is unity, i.e.,

$$
T(\theta=0) \sim \frac{\Gamma_{\uparrow} \Gamma_{\uparrow}}{\left(\epsilon-\epsilon_{o}\right)^{2}+\left(\frac{\Gamma_{\uparrow}+\Gamma_{\uparrow}}{2}\right)^{2}} \sim 1,
$$
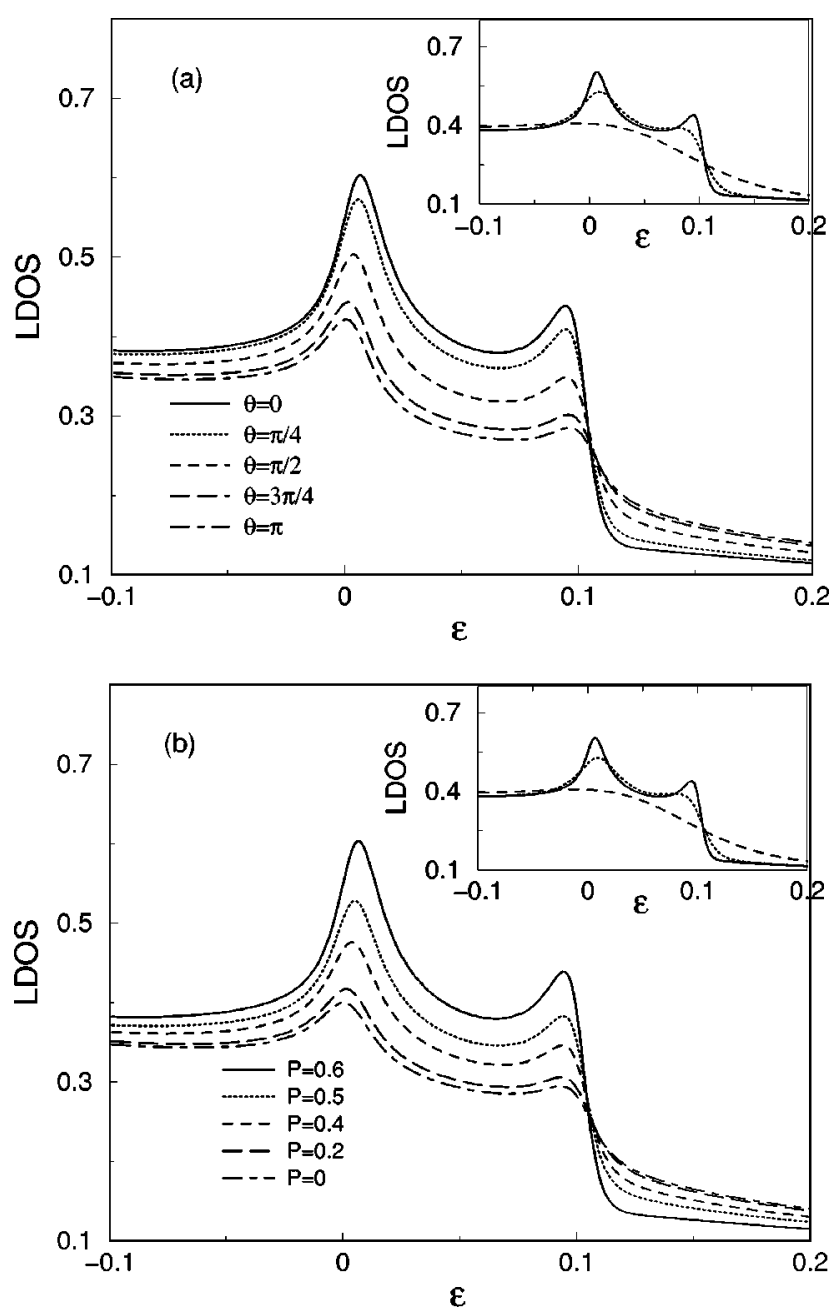

FIG. 2. (a) LDOS versus energy $\epsilon$ with on-site Coulomb interaction $U=6.0$ and chemical potentials $\mu_{L}=0.1, \mu_{R}=0.0$ for different orientation angles. Inset: LDOS versus energy for different temperatures $K_{B} T=0.005$ (solid line), $K_{B} T=0.01$ (dotted line), $K_{B} T$ $=0.05$ (dashed line) at $\theta=0$ and polarization $P=0.6$. (b) LDOS versus $\epsilon$ for different polarization. Inset: LDOS versus $\epsilon$ for different temperatures $K_{B} T=0.005$ (solid line), $K_{B} T=0.01$ (dotted line), $K_{B} T=0.05$ (dashed line) at $\theta=0$ and polarization $P=0.6$.
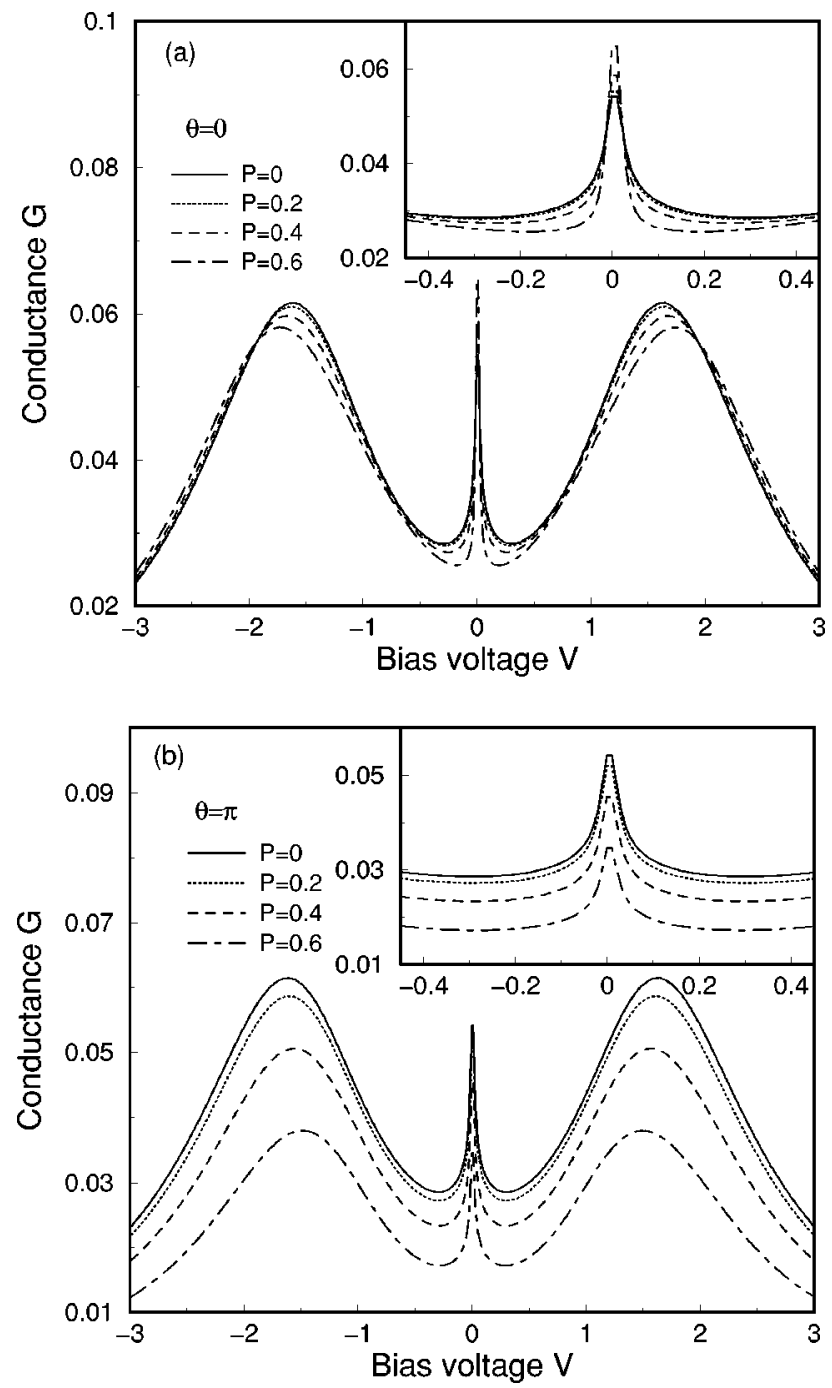

FIG. 3. Nonlinear differential conductance as a function of bias voltage at different polarization. (a) For orientation angle $\theta=0$; (b) for $\theta=\pi\left(K_{B} T=0.005\right)$. The insets in (a) and (b) show the Kondo peak region more clearly.

which is independent of $P$. Figure 3(a) shows some small changes with $P$, this is due to the effect of interaction $U$. When $U \neq 0$, the coupling parameters $\Gamma_{\sigma}$ in the above expression are "renormalized" from their bare values, therefore the $P$ dependence of the effective coupling parameters becomes more complicated. When $\theta=\pi$, the Briet-Wigner tunneling formula becomes

$$
T(\theta=\pi) \sim \frac{\Gamma_{\uparrow} \Gamma_{\downarrow}}{\left(\epsilon-\epsilon_{o}\right)^{2}+\left(\frac{\Gamma_{\uparrow}+\Gamma_{\downarrow}}{2}\right)^{2}} .
$$

It is not difficult to confirm, from the definition of $P$, that $T(\theta=\pi)$ decreases as $P$ increases which is shown in Fig. 3(b).

Although the main peaks of the interacting system can be qualitatively understood from resonance tunneling considerations, the Kondo peak appears to behave differently. In particular, our investigation found that the $P$ dependence of the 


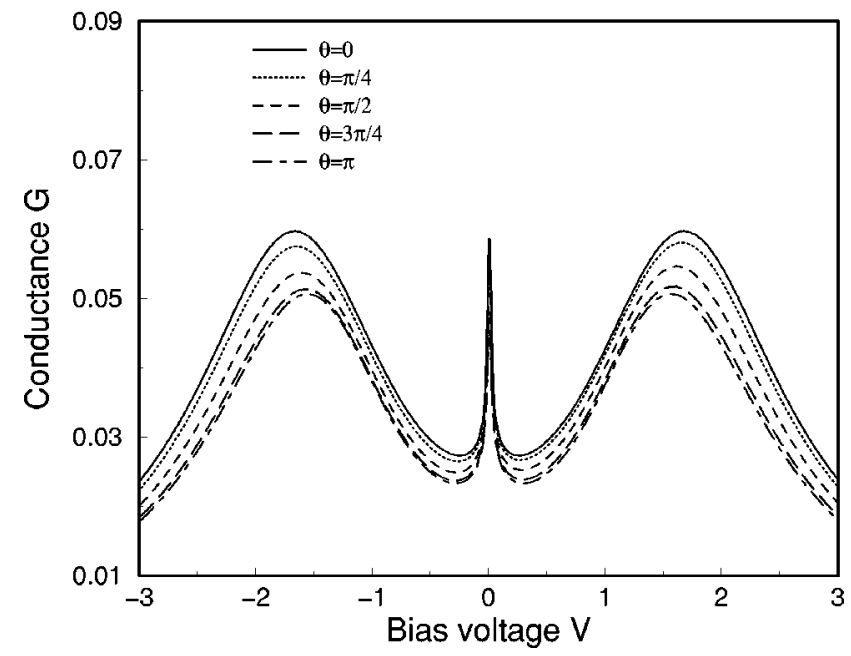

FIG. 4. The nonlinear differential conductance versus bias voltage at polarization $P=0.6$ and different orientation angles $\left(K_{B} T\right.$ $=0.005, U=6.0)$.

Kondo peak can change depending on other system parameters such as the relative position of the QD energy level $\epsilon_{0}$. At $\theta=0$, Fig. 3(a) (and its inset) shows the Kondo peak to increase with $P$; and at $\theta=\pi$, the Kondo peak tends to decrease [Fig. 3(b) and inset]. Of course, there is no apparent reason for the Kondo peak to behave the same way as the main peaks, because the physical origin of these peaks are totally different: the main peaks occur due to tunneling from the leads to the QD while Kondo peak emerges as a result of high-order co-tunneling processes in which the intermediate states are only possible for a very short time determined by the Heisenberg principle.

Next, we study the spin-valve effect by fixing polarization of the ferromagnetic leads and varying the relative orientation angle $\theta$ of the magnetic moments. Figure 4 shows conductance as a function of bias voltage for several angles $\theta$ with $P=0.6$. The Kondo peak as well as the main peaks all decrease with increasing $\theta$ and it can be explained exactly the same way as above. When $\theta$ changes from zero to $\pi$, it gives rise to a rearrangement of the number of spin-up and spin-down electrons. Therefore the couplings for spin-up and spin-down electrons become different and conductance decreases. Figures 5(a) and 5(b) show clearly how zero-bias conductance vary with $\theta$ for several values of $P$ with dot level $\epsilon_{0}=-1$ [Fig. 5(a)] and $\epsilon_{0}=-4$ [Fig. 5(b)]. These results show the familiar spin-valve behavior ${ }^{41}$ in that $G$ is modulated by the polarization angle $\theta$ even though there is a strong $e-e$ interaction $U$. Again, we note that conductance is largest at all polarizations when $\theta=0$ and smallest when $\theta$ $=\pi$. When $\epsilon_{0}=-4$, the LDOS is shifted which leads to a smaller contribution to current from tunneling processes. This is why changes in polarization affect co-tunneling significantly and conductances are separated from each other for different values of $P$.

\section{SUMMARY}

In this work, quantum transport properties of an interacting mesoscopic quantum dot connected to two metallic fer-
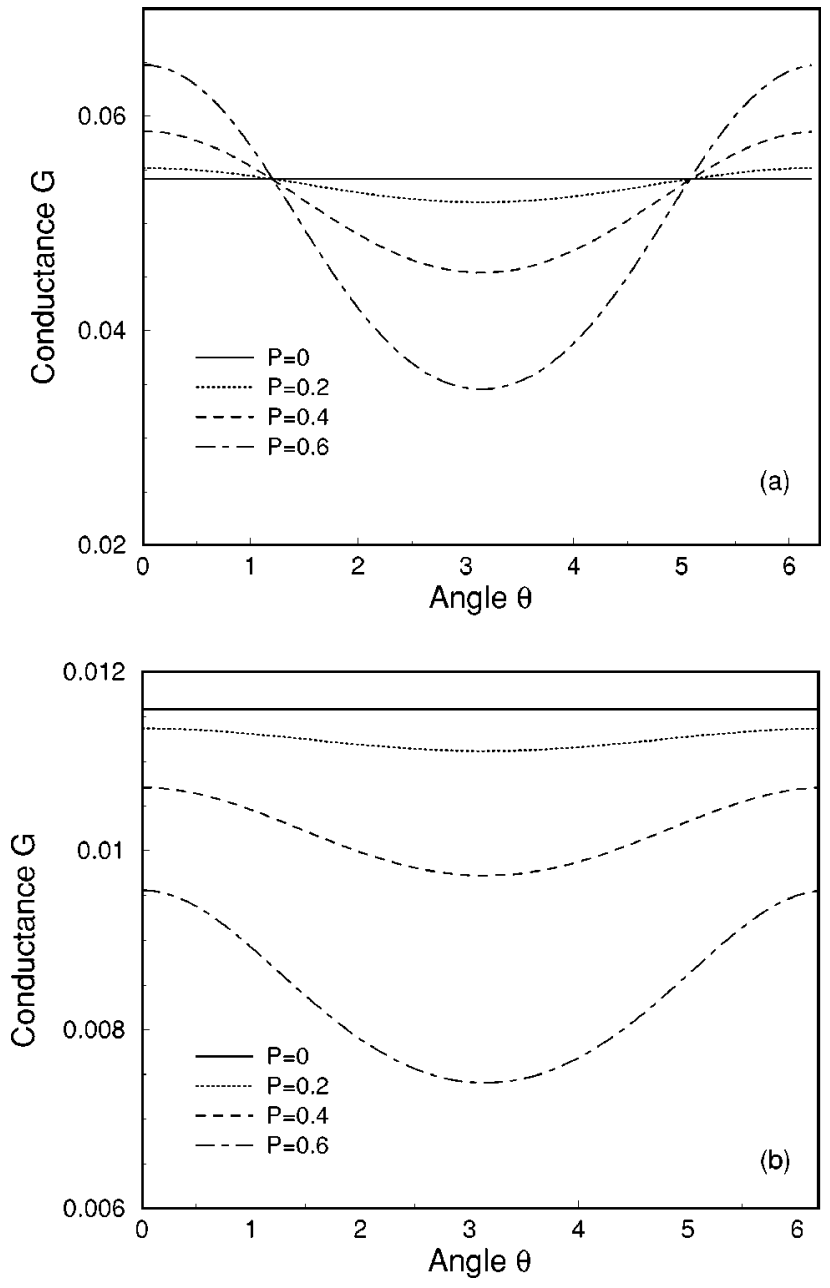

FIG. 5. Nonlinear differential conductance as a function of orientation $\theta$ for different polarizations. (a) For interdot energy level $\epsilon_{0}=-1.0$; (b) For $\epsilon_{0}=-4.0$. Other parameters are the same as those of Fig. 4.

romagnetic electrodes have been studied theoretically. Our theory was based on the Anderson model applied to spinpolarized transport through the TMR system. The presence of the strong $e-e$ interaction makes the analysis much more complicated, however, it is this interaction that is responsible for the Kondo phenomenon. Our analysis suggests that not only the resonance tunneling and the Coulomb charging peaks, but also the Kondo peak, are affected by the magnetic properties of the ferromagnetic leads. This finding indicates that in addition to the conventional spin-valve modulation of the current, the many-body physics in terms of the Kondo resonance can also be controlled by such an effect. In particular, a quantum dot based TMR device is, perhaps, a good system for experimental investigations of Kondo effect since it carries low spin-polarized current which can be varied by polarization and magnetic moment of the ferromagnetic leads. Finally, our results suggest that in the presence of strong $e-e$ interaction, the usual spin-valve effect persists in that the conductance can be controlled by the relative angle of the magnetic moments.

We gratefully acknowledge financial support from the 
Natural Science and Engineering Research Council of Canada and le Fonds pour la Formation de Chercheurs et l'Aide à la Recherche de la Province du Québec (H.G.); and for a RGC grant from the SAR Government of Hong Kong under Grant No. HKU 7215/99P (J.W.). Q.S. acknowledges financial support from the Richard H. Tomlinson Foundation.

\section{APPENDIX}

In Sec. II, Eqs. (10)-(14) have been used to solve the Green's function $\mathbf{G}^{R}$. Because of the complications brought about by parameters $\theta$ and $U$, in this Appendix we present some details concerning the derivation of the final expressions (18) and (19).

In order to solve Eq. (14) for $\left\langle\left\langle d_{\bar{\sigma}}^{+} d_{\bar{\sigma}}^{-} d_{\sigma}, d_{\sigma^{\prime}}^{+}\right\rangle\right\rangle$, one has to obtain solution for all the high-order elements included in this equation. For example, matrix element $\left\langle\left\langle c_{k L}^{+}-d_{\sigma}^{-} d_{\sigma}, d_{\sigma^{\prime}}^{+}\right\rangle\right\rangle$satisfies (from equation of motion)

$$
\begin{aligned}
(\omega+ & \left.\epsilon_{k L \bar{\sigma}}-2 \epsilon_{0}-U\right)\left\langle\left\langle c_{k L \bar{\sigma}}^{+} d_{\bar{\sigma}} d_{\sigma}, d_{\sigma^{\prime}}^{+}\right\rangle\right\rangle \\
= & -T_{k L \bar{\sigma}}^{\star}\left\langle\left\langle d_{\bar{\sigma}}^{+} d_{\bar{\sigma}}^{-} d_{\sigma}, d_{\sigma^{\prime}}^{+}\right\rangle\right\rangle \\
& +\sum_{k^{\prime}} T_{k^{\prime} L \bar{\sigma}}^{\star}\left\langle\left\langle c_{k L \bar{\sigma}}^{+} c_{k^{\prime} L \bar{\sigma}} d_{\sigma}, d_{\sigma^{\prime}}^{+}\right\rangle\right\rangle \\
& +\sum_{k^{\prime}} T_{k^{\prime} L \sigma}^{\star}\left\langle\left\langle c_{k L \bar{\sigma}}^{+} d_{\bar{\sigma}}^{-} c_{k^{\prime} L \sigma}, d_{\sigma^{\prime}}^{+}\right\rangle\right\rangle \\
& +\sum_{k^{\prime}} T_{k^{\prime} R \bar{\sigma}}^{\star} \cos \frac{\theta}{2}\left\langle\left\langle c_{k L \bar{\sigma}}^{+} c_{k^{\prime} R \bar{\sigma}} d_{\sigma}, d_{\sigma^{\prime}}^{+}\right\rangle\right\rangle \\
& -\sum_{k^{\prime}} T_{k^{\prime} R \bar{\sigma}}^{\star} \bar{\sigma} \sin \frac{\theta}{2}\left\langle\left\langle c_{k L \bar{\sigma}}^{+} c_{k^{\prime} R \sigma^{\prime}} d_{\sigma}, d_{\sigma^{\prime}}^{+}\right\rangle\right\rangle \\
& +\sum_{k^{\prime}} T_{k^{\prime} R \sigma}^{\star} \cos \frac{\theta}{2}\left\langle\left\langle c_{k L \bar{\sigma}}^{+} d_{\bar{\sigma}}^{-} c_{k^{\prime} R \sigma}, d_{\sigma^{\prime}}^{+}\right\rangle\right\rangle \\
& -\sum_{k^{\prime}} T_{k^{\prime} R \sigma}^{\star} \sigma \sin \frac{\theta}{2}\left\langle\left\langle c_{k L \bar{\sigma}}^{+} d_{\bar{\sigma}} c_{k^{\prime} R \bar{\sigma}}, d_{\sigma^{\prime}}^{+}\right\rangle\right\rangle .
\end{aligned}
$$

Using the approximation Eqs. (15)-(17), we can rewrite this equation in the following way:

$$
\begin{aligned}
& \left(\omega+\epsilon_{k L \bar{\sigma}}-2 \epsilon_{0}-U\right)\left\langle\left\langle c_{k L \bar{\sigma}}^{+} d_{\bar{\sigma}}^{-} d_{\sigma}, d_{\sigma^{\prime}}^{+}\right\rangle\right\rangle \\
& \quad=-T_{k L \bar{\sigma}}^{\star}\left\langle\left\langle d_{\bar{\sigma}}^{+} d_{\bar{\sigma}}^{-} d_{\sigma}, d_{\sigma^{\prime}}^{+}\right\rangle\right\rangle+T_{k L \bar{\sigma}}^{\star}\left\langle c_{k L \bar{\sigma}}^{+} c_{k L \bar{\sigma}}\right\rangle\left\langle\left\langle d_{\sigma}, d_{\sigma^{\prime}}^{+}\right\rangle\right\rangle .
\end{aligned}
$$

Since $\left\langle c_{k L \bar{\sigma}}^{+} c_{k L \bar{\sigma}}\right\rangle$ is just a Fermi function $f\left(\epsilon_{k L \bar{\sigma}}\right)$ of the left lead, matrix element $\left\langle\left\langle c_{k L \bar{\sigma}}^{+} d_{\bar{\sigma}}^{-} d_{\sigma}, d_{\sigma^{\prime}}^{+}\right\rangle\right\rangle$can be easily determined from Eq. (A2). All other matrix elements included in Eq. (14) can be obtained in a similar manner.

Next, we substitute these high-order matrix elements into Eq. (14). To simplify notation we introduce the following self-energies:

$$
\begin{gathered}
\Sigma_{L ; \sigma \sigma}^{0}=\sum_{k} \frac{T_{k L \sigma} T_{k L \sigma}^{*}}{\omega-\epsilon_{k L \sigma}} ; \\
\Sigma_{R ; \sigma \sigma}^{0}=\sum_{k}\left(\begin{array}{c}
\cos ^{2} \frac{\theta}{2} \\
\frac{\sin ^{2} \frac{\theta}{2}}{\omega-\epsilon_{k R \sigma}}+\frac{\epsilon_{k R \bar{\sigma}}}{\omega-\epsilon_{k R \sigma}} T_{k R \sigma}^{*} ;
\end{array}\right.
\end{gathered}
$$

$$
\begin{gathered}
\Sigma_{R ; \sigma \bar{\sigma}}^{0}=-\sum_{k}\left(\frac{\bar{\sigma} \cos \frac{\theta}{2} \sin \frac{\theta}{2}}{\omega-\epsilon_{k R \sigma}}+\frac{\sigma \cos \frac{\theta}{2} \sin \frac{\theta}{2}}{\omega-\epsilon_{k R \bar{\sigma}}}\right) T_{k R \bar{\sigma}} T_{k R \sigma}^{*} \\
\Sigma_{L ; \sigma \sigma}^{1}=\sum_{k} \frac{T_{k L \sigma} T_{k L \sigma}^{*}}{\omega+\epsilon_{k L \sigma}-2 \epsilon_{0}-U}
\end{gathered}
$$$$
\Sigma_{R ; \sigma \sigma}^{1}=\sum_{k}\left(\frac{\cos ^{2} \frac{\theta}{2}}{\omega+\epsilon_{k R \sigma}-2 \epsilon_{0}-U}\right.
$$$$
\left.+\frac{\sin ^{2} \frac{\theta}{2}}{\omega+\epsilon_{k R \bar{\sigma}}-2 \epsilon_{0}-U}\right) T_{k R \sigma} T_{k R \sigma}^{*} ;
$$$$
\Sigma_{R ; \sigma \bar{\sigma}}^{1}=-\sum_{k}\left(\frac{\bar{\sigma} \cos \frac{\theta}{2} \sin \frac{\theta}{2}}{\omega+\epsilon_{k R \sigma}-2 \epsilon_{0}-U}\right.
$$$$
\left.+\frac{\sigma \cos \frac{\theta}{2} \sin \frac{\theta}{2}}{\omega+\epsilon_{k R \bar{\sigma}}-2 \epsilon_{0}-U}\right) T_{k R \bar{\sigma}} T_{k R \sigma}^{*} ;
$$$$
\Sigma_{L ; \sigma \sigma}^{a}=\sum_{k} \frac{T_{k L \sigma} T_{k L \sigma}^{*}}{\omega+\epsilon_{k L \sigma}-2 \epsilon_{0}-U} f_{L}\left(\epsilon_{k L \sigma}\right)
$$$$
\sum_{R ; \sigma \sigma}^{a}=\sum_{k}\left(\frac{\cos ^{2} \frac{\theta}{2} f_{R}\left(\epsilon_{k R \sigma}\right)}{\omega+\epsilon_{k R \sigma}-2 \epsilon_{0}-U}\right.
$$$$
\left.+\frac{\sin ^{2} \frac{\theta}{2} f_{R}\left(\epsilon_{k R \bar{\sigma}}\right)}{\omega+\epsilon_{k R \bar{\sigma}-2} \epsilon_{0}-U}\right) T_{k R \sigma} T_{k R \sigma}^{*} ;
$$$$
\Sigma_{R ; \sigma \bar{\sigma}}^{a}=-\sum_{k}\left(\frac{\bar{\sigma} \cos \frac{\theta}{2} \sin \frac{\theta}{2} f_{R}\left(\epsilon_{k R \sigma}\right)}{\omega+\epsilon_{k R \sigma}-2 \epsilon_{0}-U}\right.
$$$$
\left.+\frac{\sigma \cos \frac{\theta}{2} \sin \frac{\theta}{2} f_{R}\left(\epsilon_{k R \bar{\sigma}}\right)}{\omega+\epsilon_{k R \bar{\sigma}}-2 \epsilon_{0}-U}\right) T_{k R \bar{\sigma}} T_{k R \sigma}^{*}
$$$$
\Sigma_{L ; \sigma \sigma}^{b}=\sum_{k} \frac{T_{k L \sigma} T_{k L \sigma}^{*}}{\omega-\epsilon_{k L \sigma}} f_{L}\left(\epsilon_{k L \sigma}\right)
$$ 


$$
\begin{aligned}
\sum_{R ; \sigma \sigma}^{b}= & \sum_{k}\left(\frac{\cos ^{2} \frac{\theta}{2} f_{R}\left(\epsilon_{k R \sigma}\right)}{\omega-\epsilon_{k R \sigma}}\right. \\
& \left.+\frac{\sin ^{2} \frac{\theta}{2} f_{R}\left(\epsilon_{k R \bar{\sigma}}\right)}{\omega-\epsilon_{k R \bar{\sigma}}}\right) T_{k R \sigma} T_{k R \sigma}^{*} ; \\
\Sigma_{R ; \sigma \bar{\sigma}}^{b}=- & \sum_{k}\left(\frac{\bar{\sigma} \cos \frac{\theta}{2} \sin \frac{\theta}{2} f_{R}\left(\epsilon_{k R \sigma}\right)}{\omega-\epsilon_{k R \sigma}}\right. \\
+ & \left.\frac{\sigma \cos \frac{\theta}{2} \sin \frac{\theta}{2} f_{R}\left(\epsilon_{k R \bar{\sigma}}\right)}{\omega-\epsilon_{k R \bar{\sigma}}}\right) T_{k R \bar{\sigma}} T_{k R \sigma}^{*} .
\end{aligned}
$$

We further define some combined self-energies,

$$
\begin{gathered}
\Sigma_{\sigma \sigma^{\prime}}^{i}=\Sigma_{L ; \sigma \sigma^{\prime}}^{i}+\Sigma_{R ; \sigma \sigma^{\prime}}^{i}, \\
\Sigma_{\alpha ; \sigma \sigma^{\prime}}^{a b}=\Sigma_{\alpha ; \sigma \sigma^{\prime}}^{a}+\Sigma_{\alpha ; \sigma \sigma^{\prime}}^{b},
\end{gathered}
$$

where $i=0,1, a, b$ and $\alpha=L, R$.

With these definitions, Eq. (14) takes the form

$$
\begin{aligned}
& \left\langle\left\langle d_{\bar{\sigma}}^{+} d_{\bar{\sigma}}^{-} d_{\sigma}, d_{\sigma}^{+}\right\rangle\right\rangle=Y_{\sigma} A\left[\left\langle n_{\bar{\sigma}}^{-}\right\rangle+\Sigma_{R ; \sigma \sigma}^{1} Y_{\bar{\sigma}}\left\langle d_{\sigma}^{+} d_{\sigma}^{-}\right\rangle\right] \\
& -Y_{\sigma} A\left[\Sigma_{\bar{\sigma} \sigma}^{a b}+\Sigma_{R ; \sigma \bar{\sigma}}^{1} Y_{\bar{\sigma}}^{-\Sigma_{R ; \bar{\sigma} \sigma}^{a b}}\right] \\
& \times\left\langle\left\langle d_{\sigma}, d_{\sigma}^{+}\right\rangle\right\rangle+Y_{\sigma} A\left[\Sigma_{R ; \sigma \bar{\sigma}}^{a b}\right. \\
& \left.+\Sigma_{R ; \sigma \sigma}^{1} Y_{\sigma}^{-} \Sigma_{\sigma \sigma}^{a b}\right]\left\langle\left\langle d_{\sigma}^{-}, d_{\sigma}^{+}\right\rangle\right\rangle \text {. }
\end{aligned}
$$

Similarly,

$$
\begin{aligned}
\left\langle\left\langle d_{\sigma}^{+} d_{\sigma} d_{\bar{\sigma}}, d_{\sigma}^{+}\right\rangle\right\rangle= & -Y_{\bar{\sigma}}^{-} A\left[\left\langle d_{\sigma}^{+} d_{\bar{\sigma}}^{-}\right\rangle+\Sigma_{R ; \overline{\sigma \sigma}}^{1} Y_{\sigma}\left\langle n_{\bar{\sigma}}^{-}\right\rangle\right] \\
& -Y_{\bar{\sigma}} A\left[\Sigma_{\sigma \sigma}^{a b}+\Sigma_{R ; \bar{\sigma} \sigma}^{1} Y_{\sigma} \Sigma_{R ; \sigma \bar{\sigma}}^{a b}\right] \\
& \times\left\langle\left\langle d_{\bar{\sigma}}^{-}, d_{\sigma}^{+}\right\rangle\right\rangle+Y_{\bar{\sigma}}^{-} A\left[\Sigma_{R ; \bar{\sigma} \sigma}^{a b}\right. \\
& \left.+\Sigma_{R ; \bar{\sigma} \sigma}^{1} Y_{\sigma} \Sigma_{\bar{\sigma} \bar{\sigma}}^{a b}\right]\left\langle\left\langle d_{\sigma}, d_{\sigma}^{+}\right\rangle\right\rangle .
\end{aligned}
$$

In these expressions we have defined the following notations:

$$
Y_{\sigma}^{-1} \equiv \omega-\epsilon_{0}-U-\Sigma_{\sigma \sigma}^{0}-\Sigma_{\bar{\sigma} \sigma}^{0}-\Sigma_{\bar{\sigma} \sigma}^{1}
$$

and

$$
A^{-1} \equiv 1-\Sigma_{R ; \sigma \bar{\sigma}}^{1} Y_{\sigma}^{-\Sigma_{R ; \bar{\sigma} \sigma}^{1} Y_{\sigma}}
$$

From Eq. (10), consider the two situations where spin indices $\sigma, \sigma^{\prime}$ have the same or opposite values. We obtain

$$
\begin{aligned}
\left(\omega-\epsilon_{0}-\Sigma_{\sigma \sigma}^{0}\right)\left\langle\left\langle d_{\sigma}, d_{\sigma}^{+}\right\rangle\right\rangle= & +\Sigma_{R ; \sigma \bar{\sigma}}^{0}\left\langle\left\langle d_{\sigma}^{-}, d_{\sigma}^{+}\right\rangle\right\rangle \\
& +U\left\langle\left\langle d_{\bar{\sigma}}^{+} d_{\bar{\sigma}}^{-} d_{\sigma}, d_{\sigma}^{+}\right\rangle\right\rangle, \\
\left(\omega-\epsilon_{0}-\Sigma_{\bar{\sigma}-\bar{\sigma}}^{0}\right)\left\langle\left\langle d_{\bar{\sigma}}, d_{\sigma}^{+}\right\rangle\right\rangle= & \Sigma_{R ; \bar{\sigma} \sigma}^{0}\left\langle\left\langle d_{\sigma}, d_{\sigma}^{+}\right\rangle\right\rangle \\
& +U\left\langle\left\langle d_{\sigma}^{+} d_{\sigma} d_{\bar{\sigma}}, d_{\sigma}^{+}\right\rangle\right\rangle .
\end{aligned}
$$

Finally, substituting Eq. (A17) into Eq. (A21), and Eq. (A18) into Eq. (A22), we obtain the following equations which can be solved to obtain the Green's functions:

$$
\begin{gathered}
B\left\langle\left\langle d_{\sigma}, d_{\sigma}^{+}\right\rangle\right\rangle=1+U Y_{\sigma} A C+D\left\langle\left\langle d_{\sigma}^{-} d_{\sigma}^{+}\right\rangle\right\rangle, \\
\bar{B}\left\langle\left\langle d_{\bar{\sigma}}, d_{\sigma}^{+}\right\rangle\right\rangle=-U Y_{\sigma^{-}} A C^{\prime}+\bar{D}\left\langle\left\langle d_{\sigma}, d_{\sigma}^{+}\right\rangle\right\rangle,
\end{gathered}
$$

where for simplicity we have defined quantities $B, C, C^{\prime}$, and $D$ as

$$
\begin{gathered}
B \equiv \omega-\epsilon_{0}-\Sigma_{\sigma \sigma}^{0}+U Y_{\sigma} A\left[\Sigma_{\bar{\sigma} \bar{\sigma}}^{a b}+\Sigma_{R ; \sigma \bar{\sigma}}^{1} Y_{\bar{\sigma}}^{\left.-\Sigma_{R ; \bar{\sigma}}^{a b}\right],}\right. \\
C \equiv\left\langle n_{\bar{\sigma}}^{-}\right\rangle+\Sigma_{R ; \sigma \bar{\sigma}}^{1} Y_{\bar{\sigma}}\left\langle d_{\sigma}^{+} d_{\bar{\sigma}}\right\rangle, \\
C^{\prime} \equiv\left\langle d_{\sigma}^{+} d_{\sigma}^{-}\right\rangle+\Sigma_{R ; \bar{\sigma} \sigma}^{1} Y_{\sigma}\left\langle n_{\sigma}^{-}\right\rangle, \\
D \equiv \Sigma_{R ; \sigma \bar{\sigma}}^{0}+U Y_{\sigma} A\left[\Sigma_{R ; \sigma \bar{\sigma}}^{a b}+\Sigma_{R ; \sigma \bar{\sigma}}^{1} Y_{\bar{\sigma}}^{\left.-\Sigma_{\sigma \sigma}^{a b}\right] .}\right.
\end{gathered}
$$

In all the equations above, $\epsilon_{0}$ is the interdot level, $U$ is the on-site Coulomb energy, $T_{k \alpha \sigma}$ is the hopping matrix between lead $\alpha$ and the quantum dot, $f_{\alpha}\left(\epsilon_{k \alpha \sigma}\right)$ is the Fermi function of electron at level $(k \sigma)$ in the lead $\alpha$.
${ }^{1}$ G. A. Prinz, Science 282, 1660 (1998).

${ }^{2}$ M. N. Baibich, J. M. Broto, A. Fert, F. Nguyen Van Dau, F. Petroff, P. Etienne, G. Creuzet, A. Friederich, and J. Chazelas, Phys. Rev. Lett. 61, 2472 (1988).

${ }^{3}$ R. Meservey and P. M. Tedrow, Phys. Rep. 238, 173 (1994).

${ }^{4}$ D. J. Monsma, J. C. Lodder, Th. J. A. Popma, and B. Dieny, Phys. Rev. Lett. 74, 5260 (1995); D. J. Monsma, R. Vlutters, and J. C. Lodder, Science 281, 407 (1998).

${ }^{5}$ S. Egger, C. H. Back, J. Krewer, and D. Pescia, Phys. Rev. Lett. 83, 2833 (1999).
${ }^{6}$ M. Dax, Semicond. Int. 20, 84 (1997).

${ }^{7}$ M. Julliere, Phys. Lett. 54A, 225 (1975).

${ }^{8}$ J. S. Moodera, L. R. Kinder, T. M. Wong, and R. Meservey, Phys. Rev. Lett. 74, 3273 (1995).

${ }^{9}$ X. Zhang, Bo-Zang Li, Gang Sun, and Fu-Cho Pu, Phys. Rev. B 56, 5484 (1997).

${ }^{10}$ A. M. Bratkovsky, Phys. Rev. B 56, 2344 (1997).

${ }^{11}$ J. Barnaś and A. Fert, Phys. Rev. Lett. 80, 1058 (1998).

${ }^{12}$ S. Zhang, P. M. Levy, A. C. Marley, and S. S. P. Parkin, Phys. Rev. Lett. 79, 3744 (1997). 
${ }^{13}$ J. Inoue and S. Maekawa, Phys. Rev. B 53, R11 927 (1996).

${ }^{14}$ J. M. MacLaren, X.-G. Zhang, and W. H. Butler, Phys. Rev. B 56, 11827 (1997).

${ }^{15}$ J. Mathon, Phys. Rev. B 56, 11810 (1997).

${ }^{16}$ Arne Brataas, Yu. V. Nazarov, J. Inoue, and Gerrit E. W. Bauer, Phys. Rev. B 59, 93 (1999).

${ }^{17}$ B. G. Wang, J. Wang, and Hong Guo, J. Phys. Soc. Jpn. 70, 2645 (2001)

${ }^{18}$ Kazuhito Tsukagoshi, Bruce W. Alphenaar, and Hiroko Ago, Nature (London) 401, 572 (1999).

${ }^{19}$ H. Mehrez, Jeremy Taylor, Hong Guo, Jian Wang, and Christopher Roland, Phys. Rev. Lett. 84, 2682 (2000).

${ }^{20}$ L. Sheng, Y. Chen, H. Y. Teng, and C. S. Ting, Phys. Rev. B 59, 480 (1999).

${ }^{21}$ L. P. Kouwenhoven and L. Glazman, Phys. World January, 33 (2001)

${ }^{22}$ Sara M. Cronenwett, Tjerk H. Oosterkamp, and Leo P. Kouwenhoven, Science 281, 540 (1998).

${ }^{23}$ Takeshi Inoshita, Science 281, 526 (1998); W. G. van der Wiel, S. De Franceschi, T. Fujisawa, J. M. Elzerman, S. Tarucha, and L. P. Kouwenhoven, ibid. 289, 2105 (2000); D. Goldhaber-Gordon, Hadas Shtrikman, D. Mahalu, David Abusch-Magder, U. Meirav, and M. A. Kastner, Nature (London) 391, 156 (1998); D. Goldhaber-Gordon, J. Göres, M. A. Kastner, Hadas Shtrikman, D. Mahalu, and U. Meirav, Phys. Rev. Lett. 81, 5225 (1998).

${ }^{24}$ S. Sasaki, S. De Franceschi, J. M. Elzerman, W. G. van der Wiel, M. Eto, S. Tarucha, and L. P. Kouwenhoven, Nature (London) 405, 764 (2000).

${ }^{25}$ Ian Affleck and Pascal Simon, Phys. Rev. Lett. 86, 2854 (2001); Mikio Eto and Yuli V. Nazarov, ibid. 85, 1306 (2000); Bogdan R. Bulka and Piotr Stefański, ibid. 86, 5128 (2001).
${ }^{26}$ L. I. Glazman and M. E. Raikh, JETP Lett. 47, 378 (1988).

${ }^{27}$ P. W. Anderson, Phys. Rev. 124, 41 (1961).

${ }^{28}$ M. A. Reed, J. N. Randall, R. J. Aggarwal, R. J. Matyi, T. M. Moore, and A. E. Wetsel, Phys. Rev. Lett. 60, 535 (1998); C. G. Smith, M. Pepper, H. Ahmed, J. E. F. Frost, D. G. Hasko, D. C. Peacock, D. A. Ritchie, and G. A. C. Jones, J. Phys. C 21, L893 (1988).

${ }^{29}$ A. T. Johnson, L. P. Kouwenhoven, W. de Jong, N. C. van der Vaart, C. J. P. M. Harmans, and C. T. Foxon, Phys. Rev. Lett. 69 , 1592 (1992).

${ }^{30}$ R. C. Ashoori, H. L. Stormer, J. S. Weiner, L. N. Pfeiffer, S. J. Pearton, K. W. Baldwin, and K. W. West, Phys. Rev. Lett. 68, 3088 (1992).

${ }^{31}$ Kicheon Kang and B. I. Min, Phys. Rev. B 52, 10689 (1995).

${ }^{32}$ The parts of the model Hamiltonian, Eqs. (3) and (4), can be deduced from a more fundamental form through a Bogoliubov transformation, see Ref. 17.

${ }^{33}$ J. C. Slonczewski, Phys. Rev. B 39, 6995 (1989).

${ }^{34}$ Antti-Pekka Jauho, Ned S. Wingreen, and Yigal Meir, Phys. Rev. B 50, 5528 (1994).

${ }^{35}$ T. K. Ng, Phys. Rev. Lett. 70, 3635 (1993).

${ }^{36}$ J. A. Appelbaum and D. R. Penn, Phys. Rev. 188, 874 (1969).

${ }^{37}$ C. Lacroix, J. Phys. F: Met. Phys. 11, 2389 (1981).

${ }^{38}$ Yigal Meir, Ned S. Wingreen, and Patrick A. Lee, Phys. Rev. Lett. 66, 3048 (1991).

${ }^{39}$ Yigal Meir, Ned S. Wingreen, and Patrick A. Lee, Phys. Rev. Lett. 70, 2601 (1993).

${ }^{40}$ T. K. Ng and P. A. Lee, Phys. Rev. Lett. 61, 1768 (1988).

${ }^{41}$ H. Jaffrès, D. Lacour, F. N. Van Dau, J. Briatico, F. Petroff, and A. Vaurès, Phys. Rev. B 64, 064427 (2001). 